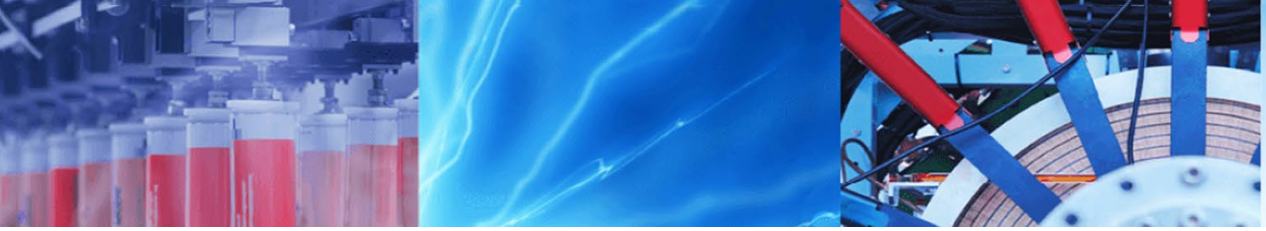

Research Article

\title{
Predictive analysis of surface roughness in argon-assisted EDM using semiempirical and ANN techniques
}

\author{
Nishant K. Singh ${ }^{1} \cdot$ Sandeep Agrawal ${ }^{1}$. Dilip Johari ${ }^{1} \cdot$ Yashvir Singh ${ }^{2}$
}

(c) Springer Nature Switzerland AG 2019

\begin{abstract}
This experimentation explores the utilization of argon-assisted electrical discharge machining (AAEDM) of high-carbon high-chromium die steel. High-pressure argon gas in conventional EDM was utilized to assess the surface roughness (SR). Analysis of variance was connected to decide the critical parameters influencing SR. In this study, a mathematical model has been instigated to get to know SR by using Buckingham pi-theorem while applying the AAEDM process. The fit summary confirmed that the quadratic model is statistically appropriate, and the lack-of-fit is insignificant. Root-mean-square error and absolute standard deviation, obtained through response surface method, were also used for developing the model and for its predicting abilities through ANN. The experiment and anticipated estimates of SR during the process, obtained by dimensional analysis and ANN, were found to be in accord with each other. However, the ANN technique proved to be more fitting to the response as compared to the dimensional analysis.
\end{abstract}

Keywords Gas pressure $\cdot$ Dimensional analysis $\cdot$ RSM $\cdot$ ANN

\section{Introduction}

Electrical discharge machining (EDM) is a material removal process and is widely used for the precision work. It has a wide range of application in mold and die making, automotive, aerospace and surgical components [1]. One of the real difficulties experienced amid EDM is flushing of debris from discharge gap. The accumulation of debris in the discharge gap results in an arcing and short circuit. This decreases a surface finish and surface integrity [2].

So as to improve machinability of EDM process, researchers explored different avenues regarding a few traditional, propelled calculation and imaginative methodologies. Because of the intricate and discretionary nature of different procedures and countless components, there is a necessity to develop most appropriate methodology. Tsai and Wang [3] utilized dimensional investigation method to decide surface roughness (SR) in various specimens made from different materials. The electrical and thermal properties of workpiece alongside significant process parameters were selected to accomplish the ideal model. The model created by the authors' was observed to be as per the test output. Yahya and Manning [4] examined the variables impacting material removal rate (MRR) amid the conventional EDM process. They distinguished critical variables through analysis of variance so as to build up a numerical model for MRR based on dimensional investigation. Kumar and Khamba [5] examined the titanium alloy specimens machined by EDM and built up a $\mu$-model to govern MRR by utilizing Buckingham pi-hypothesis approach. Patil and Brahmankar [6] come up with a mathematical model to anticipate MRR in wire electric discharge machining. They built up a model by using nonlinear estimation method as well as dimensional investigation. Bobbili et al. [7] did a similar report on wire electric discharge machining of materials used in defense for making arms by dimensional technique identified with MRR and SR. They thought about workpiece material's thermal properties,

$\triangle$ Nishant K. Singh, nishant.singh78@gmail.com | 1 Department of Mechanical Engineering, Hindustan College of Science and Technology, Mathura, Uttar Pradesh, India. ${ }^{2}$ Department of Mechanical Engineering, Sir Padampat Singhania University, Udaipur, India.

SN Applied Sciences (2019) 1:995 | https://doi.org/10.1007/s42452-019-1032-0

Received: 30 November 2018 / Accepted: 31 July 2019 / Published online: 7 August 2019 
and machine electrical parameters are key factors to build up the mathematical model for MRR and SR. Kumar et al. [8] have created numerical model to foresee TWR utilizing dimensional investigation amid powder-blended EDM of titanium composites. Their discoveries proposed that warm conductivity of example eminently influenced the TWR amid machining of cryogenic-treated workpiece.

Over the most recent couple of decades, different researchers have proposed distinctive soft computing tools to set up a relationship between machining parameters and prominent output responses like MRR and SR. Mandal et al. [9] used ANN to develop models to study the MRR and the absolute tool wear rate. Furthermore, they applied a non-dominating sorting genetic algorithm to find the optimum value of process responses. Assarzadeh and Ghoreshi [10] applied ANN to develop models and to get optimal value of responses, namely the MRR and the SR, during the EDM operation. Pradhan et al. [11] proposed two different ANN-based models for prediction of the SR during the EDM process. Their findings established that back-propagation neural network model gives more accurate results than radial basis function neural network model. Pataowari et al. [12] developed models to determine average layer thickness and material transfer rate during EDM operation by applying ANN. Kumar et al. [13] applied ANN paired with Taguchi technique for modeling and optimization of the SR. Kumar and Choudhury [14] used ANN techniques to determine the SR and wheel wear during electrical discharge diamond grinding (EDDG) of high-speed steel (HSS) specimen. They observed that ANN-based model makes more precise assessment in comparison with regression-based model. Agarwal et al. [15] developed models to determine the MRR and the SR during EDGC by applying an ANN method. Kar et al. [16] optimized the SR parameters during electro-discharge coating process by applying fuzzy logic coupled with the Taguchi technique. Prakash et al. [17] obtained optimum value of the input parameters in powder-mixed EDM by using the Taguchi-based RSM coupled with a non-dominated sorting genetic algorithm.

From the study of the previous research work, one could not find any conceivable effort on a meticulous model, in view of thermo-mechanical characteristics, which would guarantee a superior surface finish in gas-based EDM. Not very many examinations are there on the correlation of statistical and soft computing models. The greater part of the exploration has been centered on correlation of the soft computing models in EDM. Comparative investigation among semi-experimental and ANN models has not been tended to the writing.

In context of the previously mentioned predicaments, the motivation behind the current examination is to investigate and build up a model to anticipate SR by using high-pressure argon gas through multi-hole rotary tool in conventional EDM. Response surface method (RSM) based on design of experiment technique is applied for blueprints of the experimentation. Statistical analysis of the experimental data has been made during analysis of variance (ANOVA). ANOVA enables to get insight into the machining process and distinguish between the factors which have significant effects on the process responses. Based on the obtained results, ANNand semiempirical-based models have been developed to assess the effects of different machining factors on SR during argon-assisted electrical discharge machining (AAEDM) process. The efficacy of the established models in predications of machinability has been compared at the end.

\section{Experimental work}

\subsection{Electrodes material}

The test was led on D3 die steel, utilizing copper as tool material. The specimen of dimension $(20 \times 15 \times 15 \mathrm{~mm})$ was utilized for experimentation. Table 1 demonstrates the chemical constitution of the chosen workpiece.

A perforated tool was utilized to ensure that a stream of high-speed argon gas goes through the tool. So as to guarantee powerful exchange of heat from the surface of the tool, a tool having $8.35 \mathrm{~mm}$ diameter and $70 \mathrm{~mm}$ length was picked. The perforated tool is shown in Fig. 1a, and the connection utilized amid the analysis is shown in Fig. $1 \mathrm{~b}$.

\subsection{Experimental procedure}

The period of machining during the process was set at $15 \mathrm{~min}$ for each and every trial. The rotary electrode-assisted die-sinking EDM was carried out on an EDM machine (Model Smart ZNC, Electronica, India). Commercial kerosene oil was utilized as the dielectric medium. During the experiment, five procedure factors, namely discharge current, pulse-on time, duty cycle, tool speed and gas pressure were chosen. The estimation of these factors was fixed based on preliminaries experiments and machine limit. Argon gas under sufficient pressure has been utilized in pass on conventional EDM activity to keep the oxidation response, odds of flame
Table 1 Chemical composition of specimen

\section{SN Applied Sciences}

\begin{tabular}{lllllll}
\hline $\mathrm{Cr}$ & $\mathrm{C}$ & $\mathrm{Si}$ & $\mathrm{Mn}$ & $\mathrm{P}$ & $\mathrm{S}$ & $\mathrm{Fe}$ \\
\hline 10.05 & 2.30 & 0.40 & 0.30 & 0.05 & 0.03 & Rest \\
\hline
\end{tabular}




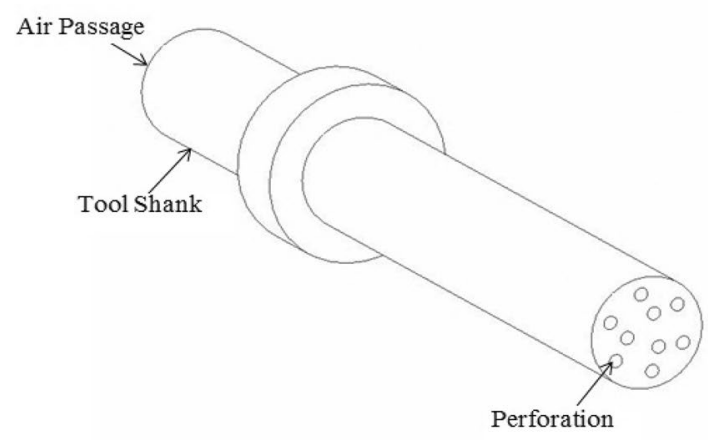

(a)

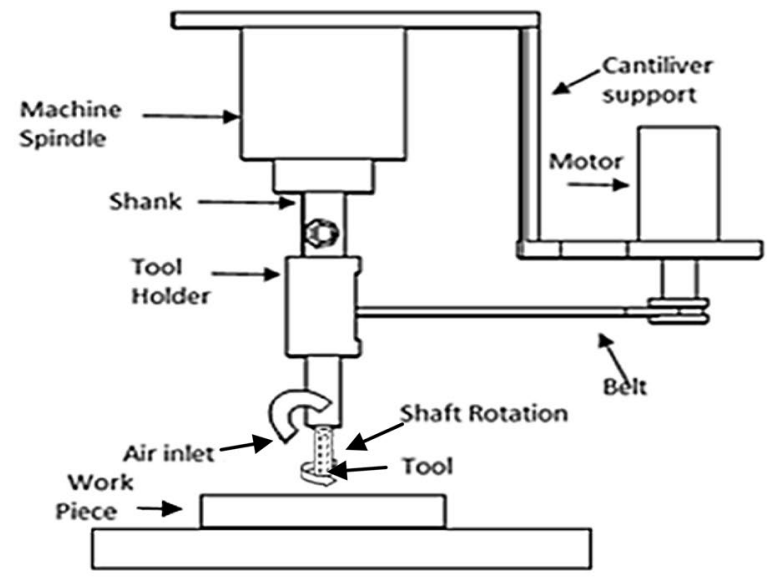

(b)

Fig. 1 Schematic images of a Tool, b Experimental setup employed [18]

Table 2 Process parameters with their range

\begin{tabular}{llllll}
\hline Parameters & \multicolumn{3}{l}{ Levels } \\
\cline { 2 - 6 } & -2 & -1 & 0 & 1 & 2 \\
\hline Discharge current $\left(I_{\mathrm{p}}\right)(\mathrm{A})$ & 3 & 4 & 5 & 6 & 7 \\
Pulse-on time $\left(T_{\text {on }}\right)(\mu \mathrm{s})$ & 100 & 200 & 300 & 400 & 500 \\
Duty cycle (DC) & 0.52 & 0.58 & 0.64 & 0.70 & 0.76 \\
Tool rotation (rpm) & 100 & 300 & 500 & 700 & 900 \\
Gas pressure (AP) $(\mathrm{mm} \mathrm{Hg})$ & 3 & 6 & 9 & 12 & 15 \\
\hline
\end{tabular}

and dangers amid the machining task. Table 2 exhibits the machining parameters run utilized for the present work. The acetone was used as cleaning agent for machined workpiece. Mitutoyo make tester was utilized to quantify the surface roughness of the machined workpiece. So as to guarantee a precise machining time computation, electronic clock (exactness of $0.1 \mathrm{~s}$ ) was utilized.

\section{Analysis of experimental data}

A total of 32 experiments were conducted using central composite rotatable design (CCRD) with independent variables at five different levels with actual units. ANOVA was performed to distinguish the essential factors affecting $S R$ amid the AAEDM procedure. The ANOVA of second-order model is given in Table 3. For the model, the estimation of 'Prob > F' is observed to be less than 0.05 (95\% certainty). Consequently, it is apparent that the factors in the model impacted the response. Equation (1) represents the regression model of SR.

$$
\begin{aligned}
\mathrm{SR}= & -5.39-\left(0.019 \times I_{\mathrm{p}}\right)-\left(0.0145 \times T_{\text {on }}\right) \\
& +(15.9 \times \mathrm{DC})+(0.000883 \times \mathrm{RPM}) \\
& +(1.16 \times \mathrm{GP})+\left(0.000956 \times I_{\mathrm{p}} \times T_{\text {on }}\right) \\
& +\left(0.0117 \times T_{\text {on }} \times \mathrm{DC}\right)-(1.76 \times \mathrm{DC} \times \mathrm{GP})
\end{aligned}
$$

\begin{tabular}{|c|c|c|c|c|c|c|c|}
\hline Source & DF & Seq.SS & MS & $F$ & $P$ & $R^{2}$ & \\
\hline Regression & 7 & 5.334 & 0.762 & 50.03 & 0 & 0.9359 & $\mathrm{~F}_{(0.05,7,24) ;}^{\text {standard }}=2.87$ \\
\hline Linear & 5 & 3.964 & & & & & $\mathrm{~F}^{\text {regression }}>F_{(0.05724}^{\text {standard }}$ \\
\hline Interaction & 2 & 1.370 & & & & & $\mathrm{~F}^{\text {standaed }}=2.91 \mathrm{~F}^{\text {lack - of }- \text { fit }}<^{\text {standard }}$ \\
\hline Residual error & 24 & 0.365 & 0.015 & & & & $\begin{array}{l}(0.05,19,24) \\
(0.05,19,24) ;\end{array}$ \\
\hline Lack-of-fit & 19 & 0.332 & & 2.68 & 0.139 & & 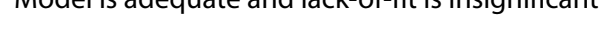 \\
\hline Pure error & 5 & 0.0015 & & & & & \\
\hline Total & 31 & 0.7332 & & & & & \\
\hline
\end{tabular}

Table 3 ANOVA table for SR 


\subsection{Dimensional analysis for evaluating the surface roughness during AAEDM process}

The dimensional analysis is a technique utilized to get specific details about a given physical problem. With the dimensional investigation, one can get a total arrangement of dimensionless parameters. The dimensional investigation technique is essentially used to lessen the multifaceted nature of a physical issue by decreasing the quantity of variables, which may not altogether influence a given issue [3]. The different attributes of physical quantities used for development of semiempirical model are given in Table 4.

On the off chance that the concerned physical issue has ' $n$ ' factors and in the event that ' $k$ ' means essential dimensions, dimensional investigation lessens the issue to just $\pi$-dimensionless terms. Normally, ' $n-k$ ' breaks even with the quantity of dimensionless $\pi$-terms that oversee the issue.

Function: $\quad \mathrm{SR}=f\left(I_{\mathrm{p}}, T_{\mathrm{on}}, \mathrm{RPM}, \mathrm{GP}, \mathrm{DC}, K, \sigma, \rho, C_{\mathrm{p}}, \theta\right)$

If the concerned physical issue has ' $n$ ' factors and if ' $k$ ' variable, in the present case, the rank of a matrix is five and there are eleven factors. Along these lines, according to Buckingham $\pi$-speculation, there are six $\pi$-terms. In like way, the measurement equation for the connection is where $Z$ is the dimensionless constant and $\alpha, \beta, \gamma, \delta$ and $\lambda$ are unidentified exponents. The nonlinear estimation method is utilized to decide the dimensionless constant and obscure exponents.

The values of $Z, \alpha, \beta, \gamma, \delta$ and $\lambda$ are found to be $23207823.51,0.1938,-0.1091,0.3412,0.0567$ and 0.3538 , respectively. Equation (4) is represented in the given form:

$$
\begin{aligned}
\mathrm{SR}= & (23207.82) X\left(\frac{I \rho C_{p}^{1.5}}{k^{1.5} \sigma^{0.5}}\right)^{0.1938} \times\left(\frac{T_{\mathrm{on}} \rho C_{\mathrm{p}}^{2} \theta}{K}\right)^{-0.1091} \\
& X\left(\frac{\mathrm{RPM} K}{\rho C_{p}^{2} \theta}\right)^{0.3412} \times\left(\frac{\mathrm{GP}}{\rho C_{p} \theta}\right)^{0.0567} X(\mathrm{DC})^{0.3538}
\end{aligned}
$$

\subsection{Prediction of SR in AAEDM by ANN}

In this work, MATLAB programming was utilized to structure the best ANN engineering. The information layer is related to discharge current, pulse-on time, duty cycle, tool speed and discharge gas pressure. The yield layer is compared to the SR. In this model, the information layer is related to a hidden layer neuron, and the hidden layer is related to yield layers. After broad preliminaries and based

$[\mathrm{L}]^{k_{1}}\left[\mathrm{QT}^{-1}\right]^{k_{2}}[\mathrm{~T}]^{k_{3}}\left[\mathrm{~T}^{-1}\right]^{k_{4}}\left[\mathrm{ML}^{-1} \mathrm{~T}^{-2}\right]^{k_{5}}\left[\mathrm{MLT}^{-3} \theta^{-1}\right]^{k_{7}}\left[\mathrm{M}^{-1} \mathrm{~L}^{-3} \mathrm{TQ}^{2}\right]^{k_{8}}\left[\mathrm{ML}^{-3}\right]^{k_{9}}\left[\mathrm{~L}^{2} \mathrm{~T}^{2} \theta^{-1}\right]^{k_{10}}[\theta]^{k_{11}}=\left[\mathrm{M}^{0} \mathrm{~L}^{0} \mathrm{~T}^{0} \theta^{0} \mathrm{Q}^{0}\right]$

$\mathrm{SR}=\left(\frac{k}{\rho C_{\mathrm{p}}^{1.5} \theta^{0.5}}\right)(Z) X\left(\frac{I \rho C_{p}^{1.5}}{k^{1.5} \sigma^{0.5}}\right)^{\alpha} \times\left(\frac{T_{\mathrm{on}} \rho C_{\mathrm{p}}^{2} \theta}{K}\right)^{\beta} \times\left(\frac{\mathrm{RPM} K}{\rho C_{\mathrm{p}}^{2} \theta}\right)^{\gamma} \times\left(\frac{\mathrm{GP}}{\rho C_{\mathrm{p}} \theta}\right)^{\delta} X(\mathrm{DC})^{\lambda}$

Table 4 Various attributes of physical quantities

\begin{tabular}{llll}
\hline Factors & Symbol & Value & Dimensions \\
\hline $\begin{array}{lll}\text { Process parameters } \\
\text { Current }\end{array}$ & $I_{\mathrm{p}}$ & & {$\left[\mathrm{QT}^{-1}\right]$} \\
$\begin{array}{l}\text { Pulse-on time } \\
\text { Rotation }\end{array}$ & $T_{\text {on }}$ & & {$[\mathrm{T}]$} \\
Gas pressure & $\mathrm{RPM}$ & & {$\left[\mathrm{T}^{-1}\right]$} \\
Duty cycle & $\mathrm{GP}$ & & {$\left[\mathrm{ML}^{-1} \mathrm{~T}^{-2}\right]$} \\
$\begin{array}{l}\text { Material properties } \\
\text { Thermal conductivity }\end{array}$ & $K$ & $50 \mathrm{~W} / \mathrm{m}-\mathrm{K}$ & {$[1]$} \\
$\begin{array}{l}\text { Electrical conductivity } \\
\text { Density }\end{array}$ & $\sigma$ & $0.01824 \mathrm{~S} / \mathrm{m}$ & {$\left[\mathrm{MLT}^{-3} \theta^{-1}\right]$} \\
Sp. Heat & $\rho$ & $7700 \mathrm{~kg} / \mathrm{m}^{3}$ & {$\left[\mathrm{ML}^{-3} \mathrm{~L}^{-3} \mathrm{TQ}^{2}\right]$} \\
Melting point & $C_{\mathrm{p}}$ & $0.46 \mathrm{Cal} / \mathrm{S} \mathrm{mole}{ }^{\circ} \mathrm{C}$ & {$\left[\mathrm{L}^{2} \mathrm{~T}^{-2} \theta^{-1}\right]$} \\
Response & $\theta$ & $14210^{\mathrm{C}}$ & {$[\theta]$} \\
Surface roughness & $\mathrm{SR}$ & & \\
\hline
\end{tabular}

on working of the system, ANN display for the SR was produced. In these models, one hidden layer consists of 15 neurons, five input and one output neurons as shown in Fig. 2. For swift and supervised learning, Levenberg-Marquardt back-propagation neural network algorithm was used during training of the network [11-13]. The network performance is measured using mean square error (MSE). MSE can be calculated as

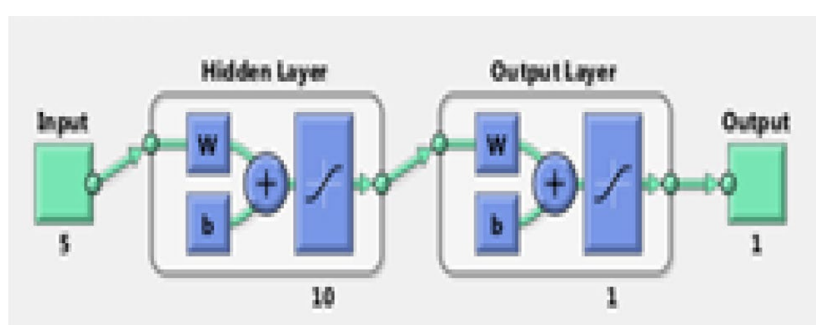

Fig. 2 ANN structure 5-15-1 [11] 
MSE $=\frac{1}{X \times Y} \sum_{i=1}^{X} \sum_{j=1}^{Y}\left(p_{j}-q_{j}\right)$

where $X$ is the number of output nodes, $Y$ is the total number of training data, $p_{j}$ is output of the $j$ th neuron and $q_{j}$ is the predicted value of $j$ th neuron [19]. Here, as per the available design matrix 32 experiment data, a total of 24 data were arbitrarily selected for the training of ANN network. The remaining eight data, which were not considered for training, were used for testing of the ANN model.

In the present ANN model for the SR during simulating, the value of correlation coefficient $(R)$ is 0.999 as shown in Fig. 3. From the statistical point of view, a network can more precisely correlate the process input to the output response if the value of the correlation coefficients is closer to 1 . Therefore, for wide range of machining conditions, a selected BP neural network effectively maps the process factors for the process output responses [9]. Figure 4 shows the comparison of actual and predicted value of the SR by FFBP-ANN. From the plot, one can observe an accord between the measured and the anticipated values as attained by the FFBP-ANN models. The accuracy of the developed model was evaluated by applying the

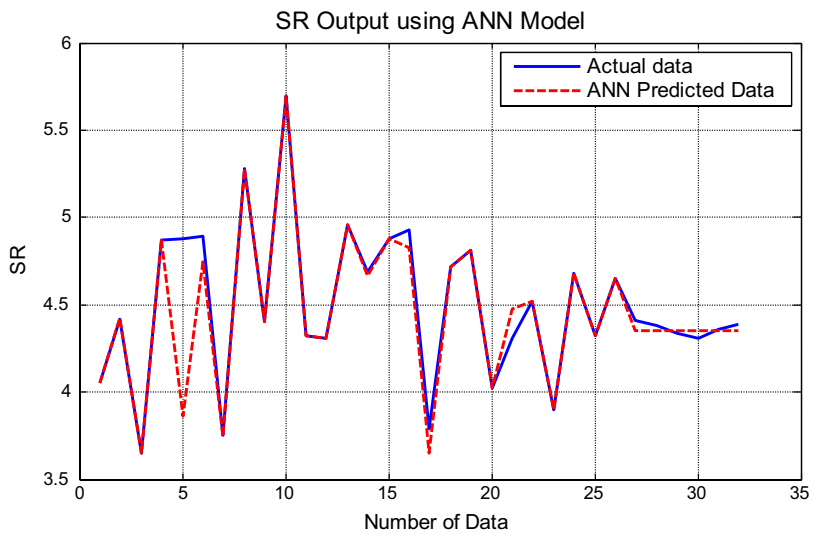

Fig. 4 Comparison of actual and predicted value SR by FFBP-ANN

root-mean-square error (RMSE) [19]. The following equation is used to obtain the RMSE.

$\operatorname{RMSE}=\sqrt{\frac{1}{T} \sum_{i=1}^{T}\left(X_{i}-Y_{i}\right)^{2}}$

where $T$ is the total training data, $X_{i}$ is the value of the measured data, and $Y_{i}$ is the value, predicted by the ANFIS model.
Fig. 3 Linear regression analysis between the experimental values and predicted values by FFBP-ANN for training, validation, testing and overall SR

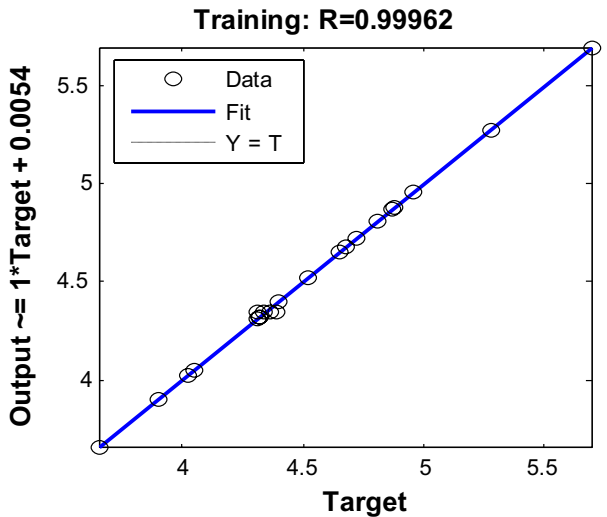

Test: $\mathbf{R}=\mathbf{0 . 5 4 6 3 4}$

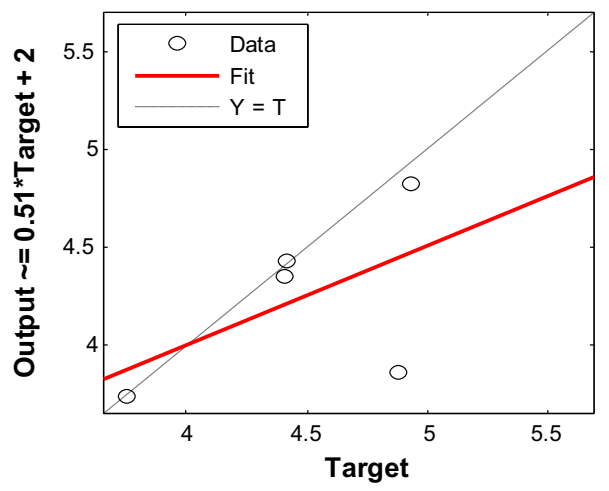

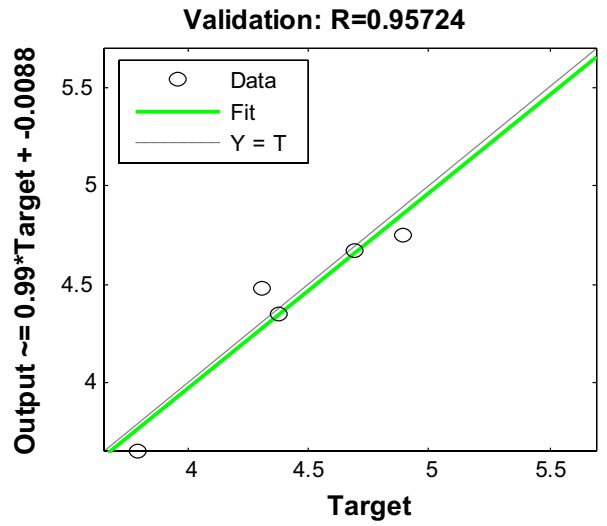

All: $\mathbf{R}=\mathbf{0 . 9 1 4 8 6}$

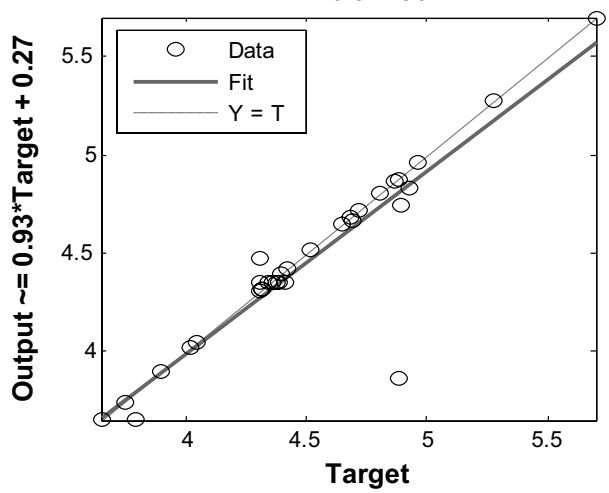

SN Applied Sciences 
Table 5 Precision of prediction models

\begin{tabular}{lllll}
\hline Parameters & Model & MSE & RMSE & $\begin{array}{l}\text { Standard } \\
\text { deviation }\end{array}$ \\
\hline & Semiempirical & $1.7227 \times 10^{-4}$ & 0.0131 & 0.4986 \\
ANFIS & $2.4414 \times 10^{-6}$ & 0.0016 & 0.0151 \\
\hline
\end{tabular}

The adequacy of the developed model was checked by the mean square error (MSE), root-mean-square error (RMSE), standard deviations and is given in Table 5. From these estimates, it can be inferred that the instigated model empowers progressively exact prediction.

\subsection{Comparison of predicted SR by ANN and Semiempirical models}

A comparison of predicted values of response by semiempirical and ANN models and measured values corresponding to each trial of the AAEDM process for the SR are shown in Table 6 . The accuracy of expectation model was assessed by using the standard deviation, MSE, RMSE and is given in Table 5. From these estimates, a conclusion may be drawn that the ANN model empowers increasingly true and exact prediction in comparison of semiempirical model. A comparison of the experiment and anticipated estimates of SR by ANN and semiempirical models is shown in Fig. 5. From figure (Refer Fig. 5), the ANN model proved to be more fitting to the response as compared to the semiempirical. The trial results and models expectation estimations of SR amid the AAEDM procedure
Table 6 Measured and predicted values of responses corresponding to each trial of AAEDM process

\begin{tabular}{|c|c|c|c|c|c|c|c|c|}
\hline Exp. no. & $I_{\mathrm{p}}$ & $T_{\text {on }}$ & DC & RPM & GP & Experiment & ANN & S-empirical \\
\hline 1 & 4 & 200 & 0.58 & 300 & 12 & 4.05 & 4.24 & 3.94 \\
\hline 2 & 6 & 200 & 0.58 & 300 & 6 & 4.44 & 4.52 & 4.38 \\
\hline 3 & 4 & 400 & 0.58 & 300 & 6 & 3.65 & 3.73 & 3.77 \\
\hline 4 & 6 & 400 & 0.58 & 300 & 12 & 4.87 & 3.67 & 3.94 \\
\hline 5 & 4 & 200 & 0.7 & 300 & 6 & 4.88 & 5.05 & 3.82 \\
\hline 6 & 6 & 200 & 0.7 & 300 & 12 & 4.89 & 4.92 & 4.32 \\
\hline 7 & 4 & 400 & 0.7 & 300 & 12 & 3.75 & 3.75 & 3.44 \\
\hline 8 & 6 & 400 & 0.7 & 300 & 6 & 5.28 & 5.13 & 3.82 \\
\hline 9 & 4 & 200 & 0.58 & 700 & 6 & 4.40 & 4.76 & 4.55 \\
\hline 10 & 6 & 200 & 0.58 & 700 & 12 & 5.70 & 5.68 & 5.62 \\
\hline 11 & 4 & 400 & 0.58 & 700 & 12 & 4.32 & 4.31 & 4.83 \\
\hline 12 & 6 & 400 & 0.58 & 700 & 6 & 4.31 & 4.41 & 5.38 \\
\hline 13 & 4 & 200 & 0.7 & 700 & 12 & 4.96 & 5.13 & 4.91 \\
\hline 14 & 6 & 200 & 0.7 & 700 & 6 & 4.69 & 4.69 & 5.46 \\
\hline 15 & 4 & 400 & 0.7 & 700 & 6 & 4.88 & 4.80 & 4.69 \\
\hline 16 & 6 & 400 & 0.7 & 700 & 12 & 4.93 & 4.93 & 4.90 \\
\hline 17 & 3 & 300 & 0.64 & 500 & 9 & 3.79 & 3.78 & 4.15 \\
\hline 18 & 7 & 300 & 0.64 & 500 & 9 & 4.72 & 4.85 & 4.79 \\
\hline 19 & 5 & 100 & 0.64 & 500 & 9 & 4.81 & 4.83 & 5.14 \\
\hline 20 & 5 & 500 & 0.64 & 500 & 9 & 4.02 & 3.98 & 4.31 \\
\hline 21 & 5 & 300 & 0.52 & 500 & 9 & 4.31 & 4.39 & 4.48 \\
\hline 22 & 5 & 300 & 0.76 & 500 & 9 & 4.52 & 4.51 & 4.32 \\
\hline 23 & 5 & 300 & 0.64 & 100 & 9 & 3.90 & 3.93 & 3.70 \\
\hline 24 & 5 & 300 & 0.64 & 900 & 9 & 4.68 & 4.68 & 5.05 \\
\hline 25 & 5 & 300 & 0.64 & 500 & 3 & 4.32 & 4.22 & 4.80 \\
\hline 26 & 5 & 300 & 0.64 & 500 & 15 & 4.65 & 4.65 & 4.46 \\
\hline 27 & 5 & 300 & 0.64 & 500 & 9 & 4.41 & 4.35 & 4.56 \\
\hline 28 & 5 & 300 & 0.64 & 500 & 9 & 4.38 & 4.35 & 4.56 \\
\hline 29 & 5 & 300 & 0.64 & 500 & 9 & 4.34 & 4.35 & 4.56 \\
\hline 30 & 5 & 300 & 0.64 & 500 & 9 & 4.31 & 4.35 & 4.56 \\
\hline 31 & 5 & 300 & 0.64 & 500 & 9 & 4.36 & 4.35 & 4.56 \\
\hline 32 & 5 & 300 & 0.64 & 500 & 9 & 4.39 & 4.35 & 4.56 \\
\hline
\end{tabular}




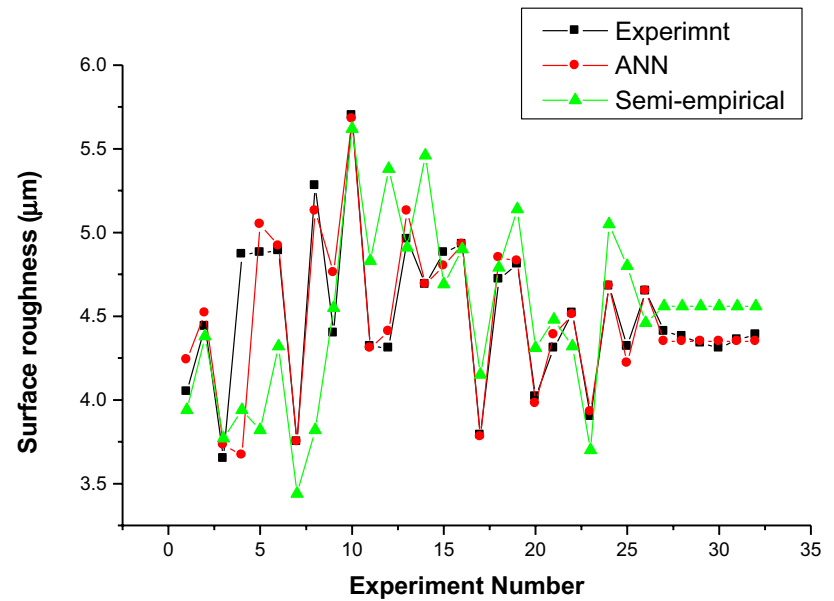

Fig. 5 Comparability of experiment and models anticipated value of SR amid AAEDM process

are observed to be as per each other. The normal error between the models forecasts, and the trial esteems are observed to be under $5 \%$.

\section{Conclusions}

This work gives insights of knowledge about the better prediction exactness in EDM process; an enhanced point of view is proposed to model SR with ANN semiempirical procedures, utilizing RSM plan of experimental strategy. The ANNand semiempirical-based methods were used to develop models for predicting the SR during the AAEDM process on high-carbon, high-chromium die steel. The following are the key findings from the study that can be summed up:

1. A semiempirical-based mathematical model was developed to predict the SR during the AAEDM process. The predicted value of responses by the model was found to be in accord with the measured value of each experiment.

2. Soft computing-based model, i.e., FFBP-ANN, was also developed for prediction of the AAEDM process performance. Due to lower values of the MSE, RMSE and standard deviation, soft computing-based model was observed to anticipate more accurately in comparison with the mathematical semiempirical model.

3. A comparison was done among the developed models to distinguish the most exact one between them. The ANN-based model outperformed the mathematical semiempirical model in general. However, ANN model was found to anticipate reactions most accurately when contrasted with semiempirical models.

\section{References}

1. Abidi MH, Al-Ahmari AM, Umer U, Rasheed MS (2018) Multiobjective optimization of micro-electrical discharge machining of nickel-titanium-based shape memory alloy using MOGA-II. Measurement 125:336-349

2. Singh NK, Pandey PM, Singh KK, Sharma MK (2016) Steps towards green manufacturing through EDM process: a review. Cogent Eng 3:1272662

3. Tsai KM, Wang PJ (2001) Semi-empirical model of surface finish on electrical discharge machining. Int J Mach Tools Manuf 41(10):1455-1477

4. Yahya A, Manning CD (2004) Determination of material removal rate of an electro-discharge machine using dimensional analysis. J Phys D Appl Phys 37(10):1467

5. Kumar J, Khamba JS (2009) Modeling the material removal rate in ultrasonic machining of titanium using dimensional analysis. Int J Adv Manuf Technol 48(1-4):103-119

6. Patil NG, Brahmankar PK (2010) Determination of material removal rate in wire electro-discharge machining of metal matrix composites using dimensional analysis. Int J Adv Manuf Technol 51(5-8):599-610

7. Bobbili R, Madhu V, Gogia AK (2015) Modelling and analysis of material removal rate and surface roughness in wire-cut EDM of armour materials. Eng Sci Technol Int J 18(4):664-668

8. Kumar S, Singh R, Batish A, Singh TP (2015) Modeling the tool wear rate in powder mixed electro-discharge machining of titanium alloys using dimensional analysis of cryogenically treated electrodes and workpiece. J Proc Mech Eng 231(2):271-282

9. Mandal D, Surjya KP, Saha P (2007) Modeling of electrical discharge machining process using back propagation neural network and multi-objective optimization using non-dominating sorting genetic algorithm-II. J Mater Proc Technol 186:154-162

10. Assarzadeh S, Ghoreishi M (2008) Neural-network-based modeling and optimization of the electro-discharge machining process. Int J Adv Manuf Technol 39:488-500

11. Pradhan MK, Das R, Biswas CK (2009) Comparisons of neural network models on surface roughness in electrical discharge machining. J Eng Manuf 223(7):801-808

12. Patowari PK, Saha P, Mishra PK (2010) Artificial neural network model in surface modification by EDM using tungsten-copper powder metallurgy sintered electrodes. Int J Adv Manuf Technol 51:627-638

13. Kumar S, Batish S, Singh R, Singh TP (2014) A hybrid Taguchiartificial neural network approach to predict surface roughness during electric discharge machining of titanium alloys. J Mech Sci Technol 28(7):2831-2844

14. Kumar S, Choudhury SK (2007) Prediction of wear and surface roughness in electro-discharge diamond grinding. J Mater Proc Technol 191:206-209

15. Agrawal SS, Yadava V (2013) Modeling and prediction of material removal rate and surface roughness in surface-electrical discharge diamond grinding process of metal matrix composites. Mater Manuf Proc 28:381-389

16. Kar S, Chakraborty S, Dey V, Ghosh SK (2017) Optimization of surface roughness parameters of Al-6351 alloy in EDC process: a taguchi coupled fuzzy logic approach. J Inst Eng India Ser 98(5):607-618. https://doi.org/10.1007/s4003 2-016-0297-y

17. Prakash C, Kansal HK, Pabla BS, Puri S (2016) Multi-objective optimization of powder mixed electric discharge machining parameters for fabrication of biocompatible layer on $\beta$-Ti alloy 
using NSGA-II coupled with Taguchi based response surface methodology. J Mech Sci Technol 30(9):4195-4204

18. Singh NK, Pandey PM, Singh KK (2016) EDM with air assisted multi-hole rotating tool. Mater Manuf Proc 31(14):1872-1878

19. Unune DR, Mali HS (2016) Artificial neural network-based and response surface methodology-based predictive models for material removal rate and surface roughness during electrodischarge diamond grinding of Inconel 718. J Eng Manuf 230(11):2082-2091. https://doi.org/10.1177/0954405415 619347

Publisher's Note Springer Nature remains neutral with regard to jurisdictional claims in published maps and institutional affiliations. 